\title{
Article \\ On the operator method for solving linear integro-differential equations with fractional conformable derivatives
}

\author{
Batirkhan Kh. Turmetov ${ }^{1, *, \ddagger}$ @0000-0001-7735-6484, Kairat I. Usmanov ${ }^{1, \ddagger} ® 0000-0002-4311-5807$ and Kulzina Zh. \\ Nazarova $^{1, \ddagger}$ (D)0000-0002-2093-1879 \\ 1 Khoja Akhmet Yassawi International Kazakh-Turkish University, Turkistan,161200, Kazakhstan; \\ batirkhan.turmetov@ayu.edu.kz; y_kairat@mail.ru;gjnazarova@mail.ru \\ * Correspondence: batirkhan.turmetov@ayu.edu.kz; \\ $\ddagger \quad$ These authors contributed equally to this work.
}

\section{check for} updates

Citation: Turmetov, B.; Usmanov, K.; Nazarova, K. On the operator method for solving linear integro-differential equations with fractional conformable derivatives. Preprints 2021, 1, 0. https://doi.org/

Received:

Accepted:

Published:

Publisher's Note: MDPI stays neutral with regard to jurisdictional claims in published maps and institutional affiliations.

\begin{abstract}
The methods for constructing solutions to integro-differential equations of the Volterra type are considered. The equations are related to fractional conformable derivatives. Explicit solutions of homogeneous and inhomogeneous equations are constructed and a Cauchy-type problem is studied. It should be noted that the considered method is based on the construction of normalized systems of functions with respect to a differential operator of fractional order.
\end{abstract}

Keywords: integro-differential equation; fractional derivatives; fractional conformable derivatives; normalized systems method.

\section{Introduction}

There are many different ways of defining fractional operators, unlike in classical calculus where there is only one way to define the derivative operation. The most common derivatives are Riemann-Liouville and Caputo derivatives, which were successfully used in modeling complex dynamical processes in physics, biology, engineering and many other fields [1-5].

Among other definitions of fractional calculus, we can mention Hilfer, Riesz, Hadamard, Erd'elyi-Kober, Atangana-Baleanu, Katugampola, fractional conformable derivatives, and many others [2], [6-9].

It should be noted that questions related to the theorems of existence and uniqueness of solutions of Cauchy-type and Dirichlet-type problems for linear and nonlinear differential equations of fractional order have been developed in sufficient detail, whereas explicit solutions are known only for certain types of linear differential equations of fractional order.

One of the most widely used methods for constructing solutions to differential equations of fractional order is the method of integral transformations. A detailed description of this method can be found in [2], [4,5] and other works. An effective method for constructing explicit solutions and solving the Cauchy problem for differential equations of fractional order is based on the Mikusinski operational calculus. In the papers of Yu. Luchko et al. [10]-[15] this method was applied to solving linear differential equations of fractional order with constant coefficients and with derivatives of Riemann-Liouville and Caputo type and the general fractional derivative. This method was later used for a general equation with a Hilfer-type operator [16]. In the paper [17] A. Pskhu formulated and solved the initial problem for linear ordinary differential equations of fractional order with Riemann-Liouville derivatives. He reduced the problem to an integral equation and constructed an explicit solution in terms of the Wright function. We also note that in $[18,19]$ the Cauchy problem for differential equations of fractional order has been studied by the Adomian decomposition method.

In this paper, we consider an operator method for constructing solutions of fractional differential equations. This method is based on the construction of normalized systems with respect to operators of fractional differentiation. The method of normalized systems was introduced in [20] and used to construct exact solutions of the Helmholtz equation 
and the polyharmonic equation. The method of normalized systems was used to solve the Cauchy problem for ordinary differential equations with constant coefficients [21], as well as to construct solutions of differential equations associated with Dunkl operators [22,23]. Later, in [24-27], this method was applied to construction of an explicit form of solution of fractional differential equations.

Let us first consider the definition of fractional-order integro-differentiation operators that will be used in this paper.

Let $0<a<b<\infty$. For function $f(x) \in C^{1}[a, b]$ we define the operator

$$
{ }_{a} T^{\alpha} f(x)=(t-a)^{1-\alpha} f^{\prime}(x), \alpha>0
$$

In case $\alpha \in(0,1)$ this operator corresponds to an integral operator of the form

$$
{ }_{a} I^{\alpha} f(x)=\int_{a}^{x} f(t) \frac{d t}{(t-a)^{1-\alpha}}
$$

Let $0<\alpha, \beta$ and ${ }_{a}^{n} T^{\alpha}=\underbrace{a^{\alpha} \cdot{ }_{a} T^{\alpha} \cdot \ldots \cdot a T^{\alpha}}_{n}, n=1,2, \ldots$. In [8], the following integrodifferential operators were considered:

$$
\begin{gathered}
{ }_{a}^{\beta} J^{\alpha} f(x)=\frac{1}{\Gamma(\beta)} \int_{a}^{x}\left(\frac{(x-a)^{\alpha}-(t-a)^{\alpha}}{\alpha}\right)^{\beta-1} f(t) \frac{d t}{(t-a)^{1-\alpha}} . \\
{ }_{a}^{\beta} D^{\alpha} f(x)=\frac{a_{a}^{n} T^{\alpha}}{\Gamma(\beta)} \int_{a}^{x}\left(\frac{(x-a)^{\alpha}-(t-a)^{\alpha}}{\alpha}\right)^{n-\beta-1} f(t) \frac{d t}{(t-a)^{1-\alpha}}, \\
{ }_{a}^{C \beta} D^{\alpha} f(x)=\frac{1}{\Gamma(\alpha)} \int_{a}^{x}\left(\frac{(x-a)^{\alpha}-(t-a)^{\alpha}}{\alpha}\right)^{n-\beta-1}{ }_{a}^{n} T^{\alpha} f(t) \frac{d t}{(t-a)^{1-\alpha}}, n-1<\beta \leq n .
\end{gathered}
$$

In case $\alpha=1$ operator ${ }_{a}^{\beta} J^{\alpha}$ coincides with the integration operator of the $\beta$ order in the Riemann-Liouville sense, whereas ${ }_{a}^{\beta} D^{\alpha}$ and ${ }_{a}^{C \beta} D^{\alpha}$ coincide with the differentiation operators of the $\beta$ order in the Riemann-Liouville and Caputo sense [2].

Further we present some well-known information about the method of normalized systems.

Let $L_{1}$ and $L_{2}$ be linear operators, acting from the functional space $X$ to $X, L_{k} X \subset$ $X, k=1,2$. Let functions from $X$ be defined in a domain $\Omega \subset R^{n}$. Let us give the definition of normalized systems [20].

Definition 1. A sequence of functions $\left\{f_{i}(x)\right\}_{i=0}^{\infty}, f_{i}(x) \in X$ is called $f$-normalized with respect to $\left(L_{1}, L_{2}\right)$ on $\Omega$, having the base $f_{0}(x)$, if on this domain the following equality holds: $L_{1} f_{0}(x)=$ $f(x), L_{1} f_{i}(x)=L_{2} f_{i-1}(x), i \geq 1$.

If $L_{2}=E$ is a unit operator, then a system of functions $f$ - normalized with respect to $\left(L_{1}, I\right)$ is called $f$ - normalized with respect to $L_{1}$, i.e. $L_{1} f_{0}(x)=f(x), L_{1} f_{i}(x)=$ $f_{i-1}(x), i \geq 1$.

If $f(x)=0$, then the system of functions $\left\{f_{i}(x)\right\}$ is just called normalized.

The main properties of the systems of functions $f$ - normalized with respect to the operators $\left(L_{1}, L_{2}\right)$ on $\Omega$ have been described in [20]. Let us consider the main property of the $f$ - normalized systems.

Proposition 1. If a system of functions $\left\{f_{i}(x)\right\}_{i=0}^{\infty}$ is $f$-normalized with respect to $\left(L_{1}, L_{2}\right)$ on $\Omega$, then the functional series $y(x)=\sum_{i=0}^{\infty} f_{i}(x), x \in \Omega$, is a formal solution of the equation: 


$$
\left(L_{1}-L_{2}\right) y(x)=f(x), x \in \Omega .
$$

The following proposition allows us to construct an $f$-normalized system with respect to a pair of operators $\left(L_{1}, L_{2}\right)$.

Proposition 2. [27] If for $L_{1}$ there exists a right inverse operator $L_{1}^{-1}$, i.e. $L_{1} \cdot L_{1}^{-1}=E$, where $E$ is a unit operator and $L_{1} f_{0}(x)=f(x)$, then a system of functions $f_{i}(x)=\left(L_{1}^{-1} \cdot L_{2}\right)^{i} f_{0}(x), i \geq 1$ is $f$-normalized with respect to a pair of operators $\left(L_{1}, L_{2}\right)$ on $\Omega$.

\section{Properties of integro-differential operators}

Let us consider some properties of operators ${ }_{a}^{\beta} J^{\alpha},{ }_{a}^{\beta} D^{\alpha}$ and ${ }_{a}^{C \beta} D^{\alpha}$.

Lemma 1. Let $\alpha, \beta>0, s>-1$ and $f(x)=(x-a)^{\alpha s}$. Then the equality holds:

$$
\left({ }_{a}^{\beta} J^{\alpha}(t-a)^{s \alpha}\right)(x)=\frac{1}{\alpha^{\beta}} \frac{\Gamma(s+1)}{\Gamma(s+1+\beta)}(x-a)^{\alpha(s+\beta)}, s>-1
$$

Proof. By the definition of the operator ${ }_{a}^{\beta} J^{\alpha}$ we have

$$
{ }_{a}^{\beta} J^{\alpha} f(x)=\frac{1}{\Gamma(\beta)} \int_{a}^{x}\left(\frac{(x-a)^{\alpha}-(t-a)^{\alpha}}{\alpha}\right)^{\beta-1}(t-a)^{s \alpha} \frac{d t}{(t-a)^{1-\alpha}} .
$$

After changing variables $\xi=\frac{(t-a)^{\alpha}}{(x-a)^{\alpha}}$ for ${ }_{a}^{\beta} J^{\alpha} f(x)$ we get

$$
\begin{gathered}
{ }_{a}^{\beta} J^{\alpha} f(x)=\frac{(x-a)^{\alpha(\beta-1)+\alpha s+\alpha}}{\alpha^{\beta} \Gamma(\beta)} \int_{0}^{1}(1-\xi)^{\beta-1} \xi^{s} d \xi=\frac{(x-a)^{\alpha s+\alpha \beta}}{\alpha^{\beta} \Gamma(\beta)} \frac{\Gamma(\beta) \Gamma(s+1)}{\Gamma(s+1+\beta)} \\
=\frac{1}{\alpha^{\beta}} \frac{\Gamma(s+1)}{\Gamma(s+1+\beta)}(x-a)^{\alpha s+\alpha \beta} .
\end{gathered}
$$

The lemma is proved.

Lemma 2. Let $f(t)=(t-a)^{\alpha s}, s>-1$. Then the following equalities hold:

$$
\begin{aligned}
& \left({ }_{a}^{\beta} D^{\alpha}(t-a)^{s \alpha}\right)(x)=\left\{\begin{array}{l}
0, s \in\{\beta-1, \beta-2, \ldots, \beta-n\} \\
\alpha^{\beta} \frac{\Gamma(s+1)}{\Gamma(s+1-\beta)}(x-a)^{\alpha(s-\beta)}, s>n-1
\end{array},\right. \\
& \left({ }_{a}^{C \beta} D^{\alpha}(t-a)^{s \alpha}\right)(x)=\left\{\begin{array}{l}
0, s \in\{0,1, \ldots, n-1\} \\
\alpha^{\beta} \frac{\Gamma(s+1)}{\Gamma(s+1-\beta)}(x-a)^{\alpha(s-\beta)}, s>n-1
\end{array},\right.
\end{aligned}
$$

Proof. If $\beta=n$ is an integer, then by definition ${ }_{a}^{\beta} D^{\alpha} f(x)={ }_{a}^{C \beta} D^{\alpha} f(x)={ }_{a}^{n} T^{\alpha}$ and thus for all $s>n-1$ we get

$$
\begin{gathered}
{ }_{a}^{n} T^{\alpha}(x-a)^{\alpha s}={ }_{a}^{n-1} T^{\alpha}\left((x-a)^{1-\alpha} \frac{d}{d x}(t-a)^{\alpha s}\right)=\alpha s \cdot{ }_{a}^{n-1} T^{\alpha}\left((x-a)^{\alpha(s-1}\right) \\
=\alpha s \cdot \alpha(s-1) \cdot \ldots \cdot \alpha(s-n+1)(x-a)^{\alpha(s-n)}=\alpha^{n} s \cdot(s-1) \cdot \ldots \cdot(s-n+1)(x-a)^{\alpha(s-n)} \\
=\alpha^{n} \frac{\Gamma(s+1)}{\Gamma(s+1-n)}(x-a)^{\alpha(s-n)} .
\end{gathered}
$$

If $s \in\{0,1, \ldots, n-1\}$, then ${ }_{a}^{n} T^{\alpha}(x-a)^{\alpha s}=0$. 
Let $n-1<\beta<n, n=1,2, \ldots$. Then, by the definition of the operator ${ }_{a}^{\beta} D^{\alpha}$ and taking into account (5) we get

$$
{ }_{a}^{\beta} D^{\alpha} f(x)={ }_{a}^{n} T^{\alpha}\left(a_{a}^{n-\beta} J^{\alpha}(t-a)^{s \alpha}\right)(x)={ }_{a}^{n} T^{\alpha}\left(\frac{1}{\alpha^{n-\beta}} \frac{\Gamma(s+1)}{\Gamma(s+1+n-\beta)}(x-a)^{\alpha(s+n-\beta)}\right) .
$$

If in the latter equality the parameter $s$ takes one of the values $s_{1}=\beta-1, s_{2}=$ $\beta-2, \ldots, s_{n}=\beta-n$, then

$$
{ }_{a}^{n} T^{\alpha}\left((x-a)^{\alpha\left(s_{j}+n-\beta\right)}\right)=0 \Rightarrow\left({ }_{a}^{\beta} D^{\alpha}(t-a)^{s_{j} \alpha}\right)(x)=0, j=1,2, \ldots, n .
$$

And if $s>\beta-1$, then

$$
\begin{gathered}
{ }_{a}^{\beta} D^{\alpha} f(x)={ }_{a}^{n} T^{\alpha}\left(\frac{1}{\alpha^{n-\beta}} \frac{\Gamma(s+1)}{\Gamma(s+1+n-\beta)}(x-a)^{\alpha(s+n-\beta)}\right) \\
=\frac{1}{\alpha^{n-\beta}} \frac{\Gamma(s+1) \alpha^{n}(s+n-\beta) \ldots(s+1-\beta)}{\Gamma(s+1+n-\beta)}(x-a)^{\alpha(s-\beta)}=\alpha^{\beta} \frac{\Gamma(s+1)}{\Gamma(s+1-\beta)}(x-a)^{\alpha(s-\beta)} .
\end{gathered}
$$

Similarly, if $s$ takes one of the values $s_{1}=0, s_{2}=1, \ldots, s_{n}=n-1$, we get

$$
{ }_{a}^{n} T^{\alpha}\left((x-a)^{\alpha s_{j}}\right)=0, j=1,2, \ldots, n \text {. }
$$

Then for these values of $s_{j}$ we get $\left({ }_{a}^{C \beta} D^{\alpha}(t-a)^{s_{j} \alpha}\right)(x)=0$.

And if $s>n-1$, then

$$
\begin{gathered}
{ }_{a}^{C \beta} D^{\alpha} f(x)={ }_{a}^{n-\beta} J^{\alpha}\left({ }_{a}^{n} T^{\alpha}(t-a)^{s \alpha}\right)(x)={ }_{a}^{n-\beta} J^{\alpha}\left(s \alpha(s \alpha-\alpha) \ldots(s \alpha-(n+1) \alpha)(x-a)^{s \alpha-n \alpha}\right)= \\
=\alpha^{n} s(s-1) \ldots(s+1-n)_{a}^{n-\beta} J^{\alpha}\left((x-a)^{\alpha(s-n)}\right)= \\
=\alpha^{n} s(s-1) \ldots(s+1-n) \frac{1}{\alpha^{n-\beta}} \frac{\Gamma(s+1-n)}{\Gamma(s+1-\beta)}(x-a)^{\alpha(s-\beta)}=\alpha^{\beta} \frac{\Gamma(s+1)}{\Gamma(s+1-\beta)}(x-a)^{\alpha(s-\beta)} .
\end{gathered}
$$

The lemma is proved.

The following assertion was proved in [8].

Lemma 3. Let $n-1<\beta \leq n, n=1,2, \ldots$ and $f(x) \in C[a, b]$. Then the equality

$$
{ }_{a}^{C \beta} D^{\alpha}\left({ }_{a}^{\beta} J^{\alpha} f(x)\right)=f(x)
$$

is valid.

\section{Construction of a solution to an integral equation}

Let $\alpha, \beta>0, m=1,2, \ldots$. Let us consider in the domain $x>a$ the following integral equation

$$
\varphi(x)=\frac{\lambda(x-a)^{\alpha \beta(m-1)}}{\Gamma(\beta)} \int_{a}^{x}\left(\frac{(x-a)^{\alpha}-(t-a)^{\alpha}}{\alpha}\right)^{\beta-1} \varphi(t) \frac{d t}{(t-a)^{1-\alpha}}+f(x) .
$$

It should be noted that for the case of the Riemann - Liouville operator, i.e. for $\alpha=1$, integral equation (9) was studied in [28]. In this work, in the case when $\alpha=1$, based on the properties of a special Mittag-Leffler type function

$$
E_{\beta, m, l}(z)=\sum_{i=0}^{\infty} c_{i} z^{i}, c_{0}=1, c_{i}=\prod_{k=0}^{i-1} \frac{\Gamma[\beta(k m+l)+1]}{\Gamma[\beta(k m+l+1)+1]}, i \geq 1
$$


an algorithm for constructing a solution to equation (9) was proposed for the cases when $f(x)$ is a polynomial or a quasi-polynomial. The properties of the function $E_{\beta, m, l}(z)$ were also studied in [29-32].

In our case, to construct a solution to equation (9), we use the method of normalized systems. For this purpose we introduce the notations $L_{1}=E$ and $L_{2}=\lambda(x-a)^{\alpha(m-1)}$. ${ }_{a}^{\beta} J^{\alpha}$, where $L_{1}$ is the unit operator. Then equation (9) can be rewritten in the form (4).

Let $f(x) \in C[a, b]$. Let us denote $\varphi_{0}(x)=f(x)$ and

$$
\varphi_{k}(x)=\left(\lambda(x-a)^{\alpha \beta(m-1)} \cdot{ }_{a}^{\beta} J^{\alpha}\right)^{k} \varphi_{0}(x), k=1,2, \ldots
$$

It is known (see, for example, [8]) that the operator ${ }_{a}^{\beta} J^{\alpha}$ is bounded from the space $C[a, b]$ to the space $C[a, b]$ and therefore for each $k=1,2, \ldots$ an inclusion $\varphi_{k}(x) \in C[a, b]$ occurs.

It is obvious that

$$
L_{1} \varphi_{0}(x)=E \varphi_{0}(x)=f(x), L_{1} \varphi_{k}(x)=\varphi_{k}(x)=L_{2}^{k} \varphi_{0}(x)=L_{2}\left(L_{2}^{k-1} \varphi_{0}(x)\right)=L_{2} \varphi_{k-1}(x)
$$

Hence, the system of functions $\varphi_{k}(x)$ from (11) is $f$-normalized with respect to the pair of operators $L_{1}=E, L_{2}=\lambda(x-a)^{\alpha(m-1)} \cdot{ }_{a}^{\beta} J^{\alpha}$.

The following assertion is valid.

Theorem 1. Let $\alpha, \beta>0, m>0, f(x) \in C[a, b]$ and $\varphi_{k}(x)$ be defined by equality (11). Then the function

$$
\varphi(x)=\sum_{k=0}^{\infty} \varphi_{k}(x)
$$

is a solution to equation (9) from class $C[a, b]$.

Proof. Let $f(x) \in C[a, b]$. Then, formally applying operators $L_{1}$ and $L_{2}$ to the series (11), we have

$$
\begin{gathered}
\left(L_{1}-L_{2}\right) \varphi(x)=L_{1} \sum_{k=0}^{\infty} \varphi_{k}(x)-L_{2} \sum_{k=0}^{\infty} \varphi_{k}(x)=f(x)+\sum_{k=1}^{\infty} \varphi_{k}(x)-\sum_{k=0}^{\infty} L_{2}^{k+1} f(x) \\
=f(x)+\sum_{k=1}^{\infty} \varphi_{k}(x)-\sum_{i=1}^{\infty} L_{2}^{i} f(x)=f(x)+\sum_{k=1}^{\infty} \varphi_{k}(x)-\sum_{i=1}^{\infty} \varphi_{i}(x)=f(x) .
\end{gathered}
$$

Hence, function $\varphi(x)$ from (12) formally satisfies equation (9). It remains to study the convergence of series (11). For this, let us estimate functions $\varphi_{k}(x)$.

For $k=1$ we get

$$
\begin{aligned}
& \left|\varphi_{1}(x)\right|=\left|\frac{\lambda(x-a)^{\alpha \beta(m-1)}}{\Gamma(\beta)} \int_{a}^{x}\left(\frac{(x-a)^{\alpha}-(t-a)^{\alpha}}{\alpha}\right)^{\beta-1} f(t) \frac{d t}{(t-a)^{1-\alpha}}\right| \\
& \leq \frac{|\lambda|(x-a)^{\alpha \beta(m-1)}}{\alpha^{\beta-1} \Gamma(\beta)} \int_{a}^{x}\left((x-a)^{\alpha}-(t-a)^{\alpha}\right)^{\beta-1}|f(t)| \frac{d t}{(t-a)^{1-\alpha}}
\end{aligned}
$$

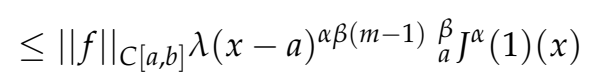

$$
\begin{aligned}
& =\|f\|_{C[a, b]}|\lambda|(x-a)^{\alpha \beta(m-1)} \frac{1}{\alpha^{\beta}} \frac{\Gamma(1)}{\Gamma(1+\beta)}(x-a)^{\alpha \beta}=\|f\|_{C[a, b]} \frac{|\lambda|}{\alpha^{\beta}} \frac{\Gamma(1)}{\Gamma(1+\beta)}(x-a)^{\alpha \beta m} \text {. }
\end{aligned}
$$


For $k=2$ we get

$$
\begin{gathered}
\left|\varphi_{2}(x)\right| \leq \frac{|\lambda|(x-a)^{\alpha \beta(m-1)}}{\Gamma(\beta)} \int_{a}^{x}\left(\frac{(x-a)^{\alpha}-(t-a)^{\alpha}}{\alpha}\right)^{\beta-1}\left|\varphi_{1}(t)\right| \frac{d t}{(t-a)^{1-\alpha}} \\
\leq\|f\|_{C[a, b]} \frac{|\lambda|^{2}}{\alpha^{\beta}} \frac{\Gamma(1)}{\Gamma(1+\beta)}(x-a)^{\alpha \beta(m-1)}\left(\begin{array}{l}
\beta \\
a
\end{array} J^{\alpha}(t-a)^{\alpha \beta m}\right)(x)= \\
=\|f\|_{C[a, b]} \frac{|\lambda|^{2}}{\alpha^{\beta}} \frac{\Gamma(1)}{\Gamma(1+\beta)}(x-a)^{\alpha \beta(m-1)} \frac{1}{\alpha^{\beta}} \frac{\Gamma(\beta m+1)}{\Gamma(\beta(m+1)+1)}(x-a)^{\alpha \beta(m+1)} \\
=\|f\|_{C[a, b]} \frac{|\lambda|^{2}}{\alpha^{2 \beta}} \frac{\Gamma(1)}{\Gamma(\beta+1)} \frac{\Gamma(\beta m+1)}{\Gamma(\beta m+\beta+1)}(x-a)^{2 \alpha \beta m} .
\end{gathered}
$$

In the general case, using the method of mathematical induction, one can prove that the inequality

$$
\left|\varphi_{k}(x)\right| \leq\|f\|_{C[a, b]}\left(\prod_{i=0}^{k-1} \frac{\Gamma[\beta i m+1]}{\Gamma[\beta(i m+1 / \beta+1)+1]}\right) \frac{|\lambda|^{k}}{\alpha^{k \beta}}(x-a)^{k \alpha \beta m}, k \geq 1
$$

is satisfied. Then

$$
\begin{gathered}
|\varphi(x)| \leq \sum_{k=0}^{\infty}\left|\varphi_{k}(x)\right| \leq|| f \|_{C}\left(1+\sum_{k=0}^{\infty}\left(\prod_{i=0}^{k-1} \frac{\Gamma[\beta i m+1]}{\Gamma[\beta(i m+1 / \beta+1)+1]}\right) \frac{|\lambda|^{k}}{\alpha^{k \beta}}(x-a)^{k \alpha \beta m}\right) \\
=\|f\|_{C[a, b]} E_{\beta, m, 1 / \beta}\left(|\lambda| \frac{(x-a)^{\alpha \beta m}}{\alpha^{\beta}}\right) .
\end{gathered}
$$

This implies absolute and uniform convergence of series (12) and the inclusion $\varphi(x) \in$ $C[a, b]$. The theorem is proved.

Now let us construct explicit solutions of Eq. (9) for particular cases of function $f(x)$

Theorem 2. Let $\alpha, \beta>0, m>0$ and $f(x)=f_{0}(x-a)^{\alpha \mu}, \mu>-1$, where $f_{0}$ is a real number. Then the solution to Eq. (9) is the function

$$
\varphi(x)=f_{0} \cdot(x-a)^{\alpha \mu} E_{\beta, m, \mu / \beta}\left(\lambda \alpha^{-\beta}(x-a)^{\alpha \beta m}\right) .
$$

Proof. Under the conditions of this theorem, system (11) can be written as

$$
\varphi_{k}(x)=\left(\lambda(x-a)^{\alpha \beta(m-1)} \cdot{ }_{a}^{\beta} J^{\alpha}\right)^{k} f_{0}(x-a)^{\mu}, k=1,2, \ldots .
$$

Find the explicit form of $\varphi_{k}(x)$. For $k=1$ we get

$$
\begin{gathered}
\varphi_{1}(x)=\lambda(x-a)^{\alpha \beta(m-1) \beta} J^{\alpha}\left(f_{0}(t-a)^{\alpha \mu}\right)(x) \\
=f_{0} \frac{\lambda(x-a)^{\alpha \beta(m-1)}}{\alpha^{\beta}} \frac{\Gamma(\mu+1)}{\Gamma(\mu+1+\beta)}(x-a)^{\alpha(\mu+\beta)}=f_{0} \frac{\lambda}{\alpha^{\beta}} \frac{\Gamma(\mu+1)}{\Gamma(\mu+1+\beta)}(x-a)^{\alpha \mu+\alpha \beta m .}
\end{gathered}
$$

For $k=2$ we get

$$
\begin{gathered}
\varphi_{2}(x)=\lambda(x-a)^{\alpha \beta(m-1)}{ }_{a}^{\beta} J^{\alpha}\left(\varphi_{1}\right)(x) \\
=\lambda(x-a)^{\alpha \beta(m-1)} f_{0} \frac{\lambda}{\alpha^{\beta}} \frac{\Gamma(\mu+1)}{\Gamma(\mu+1+\beta)}{ }_{a}^{\beta} J^{\alpha}\left((t-a)^{\alpha(\mu+\beta m)}\right)(x) \\
=(x-a)^{\alpha \beta(m-1)} f_{0} \frac{\lambda^{2}}{\alpha^{2 \beta}} \frac{\Gamma(\mu+1)}{\Gamma(\beta+\mu+1)} \frac{\Gamma(\beta m+\mu+1)}{\Gamma(\beta m+\beta+\mu+1)}(t-a)^{\alpha \mu+\alpha \beta m+\alpha \beta}
\end{gathered}
$$




$$
=f_{0} \frac{\lambda^{2}}{\alpha^{2 \beta}} \frac{\Gamma(\mu+1)}{\Gamma(\beta+\mu+1)} \frac{\Gamma(\beta m+\mu+1)}{\Gamma(\beta m+\beta+\mu+1)}(x-a)^{2 \alpha \beta m+\alpha \mu} .
$$

In the general case, for an arbitrary $k \geq 1$ we get

$$
\varphi_{k}(x)=f_{0} \frac{\lambda^{k}}{\alpha^{k \beta}}\left(\prod_{i=0}^{k-1} \frac{\Gamma[\beta(i m+\mu / \beta)+1]}{\Gamma[\beta(i m+\mu / \beta+1)+1]}\right)(x-a)^{k \alpha \beta m+\alpha \mu} .
$$

Hence, for the solution of equation (9), we obtain representation (13). The theorem is proved.

Corollary 1. Let $\alpha, \beta>0, m>0$ and $f(x)=\sum_{k=0}^{l} f_{k}(x-a)^{\alpha \mu_{k}}, \mu_{k}>-1$, where $f_{k}$ is a real number. Then the solution to equation (9) is written as

$$
\varphi(x)=\sum_{k=0}^{l} f_{k}(x-a)^{\alpha \mu_{k}} \cdot E_{\beta, m, \mu_{k} / \beta}\left(\lambda \alpha^{-\beta}(x-a)^{\alpha \beta m}\right) .
$$

Remark 1. For case $\alpha=1, a=0$ representation (14) was obtained in [28] (see Theorem 9, formula 32).

Corollary 2. Let $\alpha, \beta>0, m=1$ and $f(x)=\sum_{k=0}^{l} f_{k}(x-a)^{\alpha k}$, where $f_{k}$ is a real number. Then the solution to equation (9) is written as

$$
\varphi(x)=\sum_{k=0}^{l} k ! f_{k}(x-a)^{\alpha k} \cdot E_{\beta, k+1}\left(\lambda \alpha^{-\beta}(x-a)^{\alpha \beta}\right),
$$

where $E_{\beta, \gamma}(z)$ is a Mittag-Leffler type function [2].

\section{Construction of solutions for homogeneous fractional differential equations}

Let $\alpha>0, n-1<\beta \leq n, \gamma>0$. Let us introduce the notations ${ }_{R L} B_{\alpha, \beta}^{\gamma}=(x-$ $a)^{-\alpha \gamma}{ }_{a}^{\beta} D^{\alpha}, C_{C}^{\alpha, \beta}=(x-a)^{-\alpha \gamma}{ }_{a}^{C \beta} D^{\alpha}$. Consider in the domain $x>a$ a differential equation of the type

$$
\left(B_{\gamma}^{\alpha, \beta}-\lambda\right)^{m} y(x)=0, x>a,
$$

where $m=1,2, \ldots, B_{\gamma}^{\alpha, \beta}$ is one of the operators ${ }_{R L} B_{\alpha, \beta}^{\gamma}$ or ${ }_{C} B_{\gamma}^{\alpha, \beta}$.

Let $m=1$. If we introduce the notations $L_{1}=B_{\gamma}^{\alpha, \beta}, L_{2}=\lambda$, equation (15) can be rewritten in the form (4) and to construct a solution to this equation we have to construct a 0 - normalized system with respect to operators $\left(B_{\gamma}^{\alpha, \beta}, \lambda\right)$. In this case, we will use the method proposed in [25].

Definition 2. [25] Operator $D_{\mu}$ is called generalized - homogeneous of the $\mu$ order with respect to the variable $t$, if

$$
D_{\mu} t^{a}=C_{\mu, a} t^{a-\mu}, t>0,
$$

where $0<a \leq \mu$ is a real number, $C_{\mu, a}$ is a constant.

Let $s \in R$ and $D_{\mu}$ be a generalized - homogeneous operator of order $\mu$. Let us suppose that operator $D_{\mu}$ can be applied to the monomial $t^{\mu k+s}$. Based on equality (16), we introduce the following coefficients

$$
C(\mu, s, 0)=1, C(\mu, s, i)=\prod_{k=1}^{i}\left(t^{-\mu k-s+\mu} D_{\mu} t^{\mu k+s}\right), i \geq 1
$$


Let us assume that $C(\mu, s, i) \neq 0, i \geq 1$.

From (17) it follows that for coefficients $C(\mu, s, i)$ the equalities

$$
\frac{1}{C(\mu, s, i)}=\frac{\left(t^{-\mu i-s} D_{\mu} t^{\mu i+s+\mu}\right)}{C(\mu, s, i+1)}, i \geq 1
$$

hold.

Let $p=0,1, \ldots$. Consider the function

$$
y_{s, p}(t)=\sum_{i=p}^{\infty} \lambda^{i-p}\left(\begin{array}{c}
i \\
p
\end{array}\right) \frac{t^{\mu i+s}}{C(\mu, s, i)}
$$

where $\left(\begin{array}{c}i \\ p\end{array}\right)=\frac{i !}{p !(i-p) !}$.

Theorem 3. Let the series (18) converge and the operator $D_{\mu}$ can be applied term-by-term to it. If there exist such values of parameter s for which the equality $\left.\left(t^{-\mu i-s+\mu} D_{\mu} t^{\mu i+s}\right)\right|_{i=0}=0$, then functions $y_{s, p}(t)$ for all such values of parameters and for all $p=0,1, \ldots, m-1$ satisfy the equation

$$
\left(D_{\mu}-\lambda\right)^{m} y(t)=0, t>0, m=1,2, \ldots
$$

From equalities (6) and (7) it follows that operators ${ }_{R L} B_{\alpha, \beta}^{\gamma}$ and ${ }_{C} B_{\gamma}^{\alpha, \beta}$ are generalized homogeneous of order $\alpha(\beta+\gamma)$ with respect to $(x-a)$. Let us construct function (18) for these operators.

Consider first the case for operator ${ }_{C} B_{\gamma}^{\alpha, \beta}$. Let $\alpha>0, n-1<\beta \leq n, s_{j}=j, j=$ $0,1, \ldots, n-1$ and $f_{k, j}(x)=(x-a)^{\alpha(\beta+\gamma) k+\alpha s_{j}}, k=0,1, \ldots$

As in case (17), consider the coefficients

$$
\begin{gathered}
C\left(\alpha(\beta+\gamma), s_{j}, 0\right)=1, \\
C\left(\alpha(\beta+\gamma), s_{j}, i\right)=\prod_{k=1}^{i}\left((x-a)^{-\alpha(\beta+\gamma) k-\alpha s_{j}+a(\beta+\gamma) \beta}{ }_{a}^{\beta} D^{\alpha} f_{k, j}(x)\right), i \geq 1 .
\end{gathered}
$$

By virtue of equality (7) for $k \geq 1$, we get

$$
(x-a)^{-\alpha \gamma}{ }_{a}^{C \beta} D^{\alpha} f_{k, j}(x)=\alpha^{\beta} \frac{\Gamma\left((\beta+\gamma) k+s_{j}+1\right)}{\Gamma\left((\beta+\gamma) k+s_{j}+1-\beta\right)}(x-a)^{\alpha(\beta+\gamma) k+\alpha s_{j}-\alpha(\beta+\gamma)}, k \geq 1 .
$$

Hence,

$$
C\left(\alpha(\beta+\gamma), s_{j}, i\right)=\alpha^{i \beta} \prod_{k=1}^{i}\left(\frac{\Gamma\left((\beta+\gamma) k+s_{j}+1\right)}{\Gamma\left((\beta+\gamma) k+s_{j}+1-\beta\right)}\right) .
$$

By analogy with (18), we construct the functions

$$
\begin{gathered}
y_{j}(x)=\sum_{i=0}^{\infty} \lambda^{i} \frac{(x-a)^{\alpha(\beta+\gamma) i+s_{j} \alpha}}{C\left(\alpha(\beta+\gamma), s_{j}, i\right)}, \\
y_{j, p}(x)=\sum_{i=p}^{\infty} \lambda^{i-p}\left(\begin{array}{c}
i \\
p
\end{array}\right) \frac{(x-a)^{\alpha(\beta+\gamma) i+s_{j} \alpha}}{C\left(\alpha(\beta+\gamma), s_{j}, i\right)}, j=1,2, \ldots, n .
\end{gathered}
$$

Further, as

$$
(\beta+\gamma) k+s_{j}+1=\beta\left[\left(1+\frac{\gamma}{\beta}\right) k+\frac{s_{j}}{\beta}\right]+1=\beta\left[\left(1+\frac{\gamma}{\beta}\right)(k-1)+\frac{s_{j}+\gamma}{\beta}+1\right]+1
$$


and

$$
(\beta+\gamma) k+s_{j}+1-\beta=\beta\left[\left(1+\frac{\gamma}{\beta}\right) k+\frac{s_{j}-\beta}{\beta}\right]+1=\beta\left[\left(1+\frac{\gamma}{\beta}\right)(k-1)+\frac{s_{j}+\gamma}{\beta}\right]+1
$$

then introducing notations $m=1+\frac{\gamma}{\beta}, \ell=\frac{s_{j}+\gamma}{\beta}$ for coefficients $\frac{1}{C\left(\alpha(\beta+\gamma), s_{j}, i\right)}$ we get:

$$
\begin{gathered}
\frac{1}{C\left(\alpha(\beta+\gamma), s_{j}, i\right)}=\frac{1}{\alpha^{i \beta}} \prod_{k=1}^{i}\left(\frac{\Gamma\left[(\beta+\gamma) k+s_{j}+1-\beta\right]}{\Gamma\left[(\beta+\gamma) k+s_{j}+1\right]}\right) \\
=\frac{1}{\alpha^{i \beta}} \prod_{k=1}^{i}\left(\frac{\Gamma[\beta(m(k-1)+\ell)+1]}{\Gamma[\beta(m(k-1)+\ell+1)+1]}\right)
\end{gathered}
$$

If now we change the index $k$ to $k+1$, we finally obtain the equality

$$
\frac{1}{C\left(\alpha(\beta+\gamma), s_{j}, i\right)}=\frac{1}{\alpha^{i \beta}} \prod_{k=0}^{i-1}\left(\frac{\Gamma[\beta(m k+\ell)+1]}{\Gamma[\beta(m k+\ell+1)+1]}\right), m=1+\frac{\gamma}{\beta}, \ell=\frac{s_{j}+\gamma}{\beta}
$$

Hence, function $y_{j}(x)$ in (20) satisfies the representation

$$
y_{j}(x)=\sum_{i=0}^{\infty} \lambda^{i} \frac{(x-a)^{\alpha(\beta+\gamma) i+s_{j} \alpha}}{C\left(\alpha(\beta+\gamma), s_{j}, i\right)}=(x-a)^{s_{j} \alpha} E_{\beta, 1+\frac{\gamma}{\beta}, \frac{s_{j}+\gamma}{\beta}}\left(\lambda \alpha^{-\beta}(x-a)^{\alpha(\beta+\gamma)}\right) .
$$

Thus, the following assertion is valid.

Theorem 4. Let $\alpha>0, n-1<\beta \leq n, \gamma \geq 0, m=1, s_{j}=j, j=0,1, \ldots, n-1$. Then in case of operator $B_{\gamma}^{\alpha, \beta}={ }_{C} B_{\gamma}^{\alpha, \beta}$ solutions of equation (15) are the functions

$$
y_{j}(x)=(x-a)^{\alpha j} E_{\beta, 1+\frac{\gamma}{\beta}, \frac{j+\gamma}{\beta}}\left(\lambda \alpha^{-\beta}(x-a)^{\alpha(\beta+\gamma)}\right), j=0,1, \ldots, n-1 .
$$

We can similarly transform the functions $y_{j, p}(x)$ from (21). We get

$$
\begin{gathered}
y_{j, p}(x)=\sum_{i=p}^{\infty} \lambda^{i-p}\left(\begin{array}{c}
i \\
p
\end{array}\right) \frac{1}{\alpha^{i} \beta} \prod_{k=0}^{i-1}\left(\frac{\Gamma[\beta(m k+\ell)+1]}{\Gamma[\beta(m k+\ell+1)+1]}\right)(x-a)^{\alpha(\beta+\gamma) i+s_{j} \alpha} \\
=(x-a)^{j \alpha} \sum_{n=0}^{\infty} \lambda^{n}\left(\begin{array}{c}
n+p \\
p
\end{array}\right) \frac{1}{\alpha^{(n+p) \beta}} \prod_{k=0}^{n+p-1}\left(\frac{\Gamma[\beta(m k+\ell)+1]}{\Gamma[\beta(m k+\ell+1)+1]}\right)(x-a)^{\alpha(\beta+\gamma)(n+p)} \\
=\frac{(x-a)^{\alpha(\beta+\gamma) p+j \alpha}}{\alpha^{p \beta}} \sum_{n=0}^{\infty} \frac{(p+1)_{n}}{n !} \prod_{k=0}^{n+p-1}\left(\frac{\Gamma[\beta(m k+\ell)+1]}{\Gamma[\beta(m k+\ell+1)+1]}\right)\left(\lambda \alpha^{-\beta}(x-a)^{\alpha(\beta+\gamma)}\right)^{n} \\
=\frac{(x-a)^{\alpha(\beta+\gamma) p+j \alpha}}{\alpha^{p \beta}} E_{\beta, 1+\gamma / \beta,(j+\gamma) / \beta}^{p+1}\left(\lambda \alpha^{-\beta}(x-a)^{\alpha(\beta+\gamma)}\right),
\end{gathered}
$$

where function $E_{\beta, m, \ell}^{p+1}(z)$ is defined by the equality

$$
E_{\beta, m, \ell}^{p+1}(z)=\sum_{n=0}^{\infty} \frac{(p+1)_{n}}{n !} c_{n+p} z^{n}, c_{0}=1, c_{n}=\prod_{k=0}^{n-1} \frac{\Gamma[\beta(k m+l)+1]}{\Gamma[\beta(k m+l+1)+1]}, n \geq 1 .
$$


Theorem 5. Let $\alpha>0, n-1<\beta \leq n, \gamma \geq 0, m=1,2, \ldots, s_{j}=j, j=0,1, \ldots, n-1$. Then in case of operator $B_{\gamma}^{\alpha, \beta}={ }_{C} B_{\gamma}^{\alpha, \beta}$ solutions to equation (15) are the functions

$y_{j, p}(x)=\frac{(x-a)^{\alpha(\beta+\gamma) p+j \alpha}}{\alpha^{p \beta}} E_{\beta, 1+\gamma / \beta,(j+\gamma) / \beta}^{p+1}\left(\lambda \alpha^{-\beta}(x-a)^{\alpha(\beta+\gamma)}\right), j=0,1, \ldots, n-1, p=0,1, \ldots, m-$

Remark 2. Note that for $p=0$ we get $E_{\beta, m, \ell}^{1}(z)=E_{\beta, m, \ell}(z)$. In addition, the equality holds:

$$
\frac{1}{p !} \frac{\partial^{p}}{\partial \lambda^{p}} E_{\beta, m, \ell}(\lambda z)=z^{p} E_{\beta, m, \ell}^{p+1}(z)
$$

Solutions to differential equations with the operator ${ }_{R L} B_{\alpha, \beta}^{\gamma}=(x-a)^{-\alpha \gamma}{ }_{a}^{\beta} D^{\alpha}$ are constructed in a similar way.

The following assertion is valid.

Theorem 6. Let $\alpha>0, n-1<\beta \leq n, \gamma \geq 0, m=1,2, \ldots, s_{j}=\beta-j, j=1,2, \ldots, n$. Then in case of operator $R_{L} B_{\alpha, \beta}^{\gamma}=(x-a)^{-\alpha \gamma}{ }_{a}^{\beta} D^{\alpha}$ solutions to equation (15) are the functions

$$
y_{j, p}(x)=\frac{(x-a)^{\alpha(\beta+\gamma) p+(\beta-j) \alpha}}{\alpha^{p \beta}} E_{\beta, 1+\gamma / \beta,(\gamma+\beta-j) / \beta}^{p+1}\left(\lambda \alpha^{-\beta}(x-a)^{\alpha(\beta+\gamma)}\right),
$$

$j=1,2, \ldots, n, p=0,1, \ldots, m-1$.

Remark 3. For case $\alpha=1, a=0$ this theorem was proved in [32].

\section{Construction of solutions to inhomogeneous differential equations of fractional order}

In this section, we consider a method for constructing a solution to inhomogeneous differential equations of fractional order with operators ${ }_{a}^{\beta} D^{\alpha}$ and ${ }_{a}^{C \beta} D^{\alpha}$.

Let $\alpha>0, n-1<\beta \leq n, \gamma, v \geq 0$. Consider the equation

$$
{ }_{a}^{C \beta} D^{\alpha} y(x)=\lambda(x-a)^{\alpha \gamma v} J^{\alpha} y(x)+f(x), a<x .
$$

Let us introduce the notations $L_{1}={ }_{a}^{C \beta} D^{\alpha}, L_{2}=\lambda(x-a)^{\alpha \gamma} \cdot{ }_{a}^{v} J^{\alpha}$. Then equation (23) can be rewritten in the form (4).

First, we construct a solution to the homogeneous equation. To do this, we will construct 0 - normalized systems with respect to the pair of operators $\left({ }_{a}^{C \beta} D^{\alpha}, \lambda(x-a)^{\alpha \gamma} \cdot{ }_{a}^{v} J^{\alpha}\right)$. From proposition 2 it follows that for this purpose it is necessary to find all solutions of the equation ${ }_{a}^{C \beta} D^{\alpha} y(x)=0$ and the right inverse for the operator ${ }_{a}^{C}{ }^{\beta} D^{\alpha}$. By the proposition of Lemma 3, the right inverse to the operator ${ }_{a}^{C \beta} D^{\alpha}$ is the operator ${ }_{a}^{\beta} D^{\alpha}$, and by virtue of equality (8), linearly independent solutions of the equation ${ }_{a}^{C \beta} D^{\alpha} y(t)=0$ are functions $(x-a)^{\alpha j}, j=0,1, \ldots, n-1$.

Let $f_{0, j}(x)=(x-a)^{\alpha j}, j=0,1, \ldots, n-1$. Consider a system of functions

$$
f_{i, j}(x)=\left({ }_{a}^{\beta} J^{\alpha} \cdot \lambda(x-a)^{\alpha \gamma} \cdot{ }_{a}^{v} J^{\alpha}\right)^{i} f_{0, j}(x), i=1,2, \ldots
$$

Let us find an explicit form of the system of functions $f_{i, j}(x)$.

The following assertion is valid.

Lemma 4. For functions $f_{i, j}(x)$ equalities hold

$$
f_{i, j}(x)=\frac{1}{\alpha^{i(\beta+v)}} C_{\beta, v}(\beta+v+\gamma, j, i)(x-a)^{\alpha(\beta+v+\gamma) i+\alpha j}, i=1,2, \ldots,
$$


where

$$
\begin{aligned}
C_{\beta, v}(\beta+v+\gamma, j, i)=\prod_{k=0}^{i-1} \frac{\Gamma[(\beta+v+\gamma) k+j+1]}{\Gamma[(\beta+v+\gamma) k+j+1+v]} & \\
& \times \frac{\Gamma[(\beta+v+\gamma)(k+1)+j+1-\beta]}{\Gamma[(\beta+v+\gamma)(k+1)+j+1]} .
\end{aligned}
$$

Proof. By virtue of equality (8) we get

$$
{ }_{a}^{v} J^{\alpha}(x-a)^{\alpha j}=\frac{1}{\alpha^{v}} \frac{\Gamma(j+1)}{\Gamma(j+1+v)}(x-a)^{\alpha j+\alpha v} .
$$

Hence, for function $f_{1, j}(x)$ we obtain

$$
\begin{gathered}
f_{1, j}(x)=\left({ }_{a}^{\beta} J^{\alpha} \cdot \lambda(x-a)^{\alpha \gamma} \cdot{ }_{a}^{v} J^{\alpha}\right) f_{0, j}(x)=\frac{\lambda}{\alpha^{v}} \frac{\Gamma(j+1)}{\Gamma(j+1+v)}{ }_{a}^{\beta} J^{\alpha}(x-a)^{\alpha(j+v+\gamma)} \\
=\frac{\lambda}{\alpha^{v+\beta}} \frac{\Gamma(j+1)}{\Gamma(j+1+v)} \frac{\Gamma(v+\gamma+j+1)}{\Gamma(\beta+\gamma+j+1+v)}(x-a)^{\alpha(j+v+\gamma+\beta)} \\
=\frac{\lambda}{\alpha^{v+\beta}} \frac{\Gamma(j+1)}{\Gamma(j+1+v)} \frac{\Gamma((\beta+\gamma+v)+j+1-\beta)}{\Gamma((\beta+\gamma+v)+j+1)}(x-a)^{\alpha(\beta+v+\gamma+j)} \\
=\frac{\lambda}{\alpha^{v+\beta}} C_{\beta, v}(\beta+\gamma+v, j, 1)(x-a)^{\alpha(\beta+\gamma+v+j)}
\end{gathered}
$$

If $i=2$, then

$$
\begin{gathered}
f_{1, j}(x)=\left({ }_{a}^{\beta} J^{\alpha} \cdot \lambda(x-a)_{a}^{\alpha \gamma v} J^{\alpha}\right)^{2} f_{0, j}(x) \\
=\frac{\lambda}{\alpha^{v+\beta}} C_{\beta, v}(\beta+v+\gamma, j, 1)\left({ }_{a}^{\beta} J^{\alpha} \cdot \lambda(x-a)^{\alpha \gamma \gamma}{ }_{a}^{\alpha} J^{\alpha}\right)(x-a)^{\alpha(\beta+v+\gamma+j)} \\
=\frac{\lambda^{2}}{\alpha^{2 v+\beta}} C_{\beta, v}(\beta+v+\gamma, j, 1) \frac{\Gamma(\beta+v+\gamma+j+1)}{\Gamma(\beta+v+\gamma+j+1+v)}{ }_{a}^{\beta} J^{\alpha}(x-a)^{\alpha(\beta+2 v+2 \gamma+j)} \\
=\frac{\lambda^{2}}{\alpha^{2(v+\beta)}} C_{\beta, v}(\beta+v+\gamma, j, 1) \frac{\Gamma(\beta+v+\gamma+j+1)}{\Gamma(\beta+2 v+\gamma+j+1)} \frac{\Gamma(\beta+2 v+2 \gamma+j+1)}{\Gamma(2(\beta+v+\gamma)+j+1+v)} \\
\cdot(x-a)^{\alpha(2 \beta+2 v+2 \gamma+j)}=\frac{\lambda^{2}}{\alpha^{2(v+\beta)}} C_{\beta, v}(\beta+v+\gamma, j, 1) \frac{\Gamma((\beta+v+\gamma)+j+1))}{\Gamma((\beta+v+\gamma)+j+1+v)} \\
\cdot \frac{\Gamma(2(\beta+v+\gamma)+j+1-\beta)}{\Gamma(2(\beta+v+\gamma)+j+1)}(x-a)^{2 \alpha(\beta+v+\gamma)+\alpha j} \\
=\frac{\lambda^{2}}{\alpha^{2(v+\beta)}} C_{\beta, v}(\beta+v+\gamma, j, 2)(x-a)
\end{gathered}
$$

Further, let equality (25) hold for a natural number $r$. Then for $r+1$, we get

$$
\begin{gathered}
f_{r+1, j}(x)=\left({ }_{a}^{\beta} J^{\alpha} \cdot \lambda(x-a)^{\alpha \gamma} \cdot{ }_{a}^{v} J^{\alpha}\right)^{r+1} f_{0, j}(x)=\left({ }_{a}^{\beta} J^{\alpha} \cdot \lambda(x-a)^{\alpha \gamma} \cdot v J^{\alpha}\right) \\
\cdot\left({ }_{a}^{\beta} J^{\alpha} \cdot \lambda(x-a)^{\alpha \gamma} \cdot{ }_{a}^{v} J^{\alpha}\right)^{r} f_{0, j}(x)=\frac{\lambda^{r}}{\alpha^{r(v+\beta)}} C_{\beta, v}(\beta+v+\gamma, j, r)\left({ }_{a}^{\beta} J^{\alpha} \cdot \lambda(x-a)^{\alpha \gamma} \cdot{ }_{a}^{v} J^{\alpha}\right) \\
\cdot(x-a)^{\alpha(\beta+v+\gamma) r+\alpha j}=\frac{\lambda^{r+1}}{\alpha^{r(v+\beta)+v}} C_{\beta, v}(\beta+v+\gamma, j, r) \frac{\Gamma[(\beta+v+\gamma) r+j+1]}{\Gamma[(\beta+v+\gamma) r+j+1+v]} \\
\cdot_{a}^{\beta} J^{\alpha}(x-a)^{\alpha(\beta+v+\gamma) r+\alpha j+\alpha v+\alpha \gamma}=\frac{\lambda^{r+1}}{\alpha^{(r+1)(v+\beta)}} C_{\beta, v}(\beta+v+\gamma, j, r)
\end{gathered}
$$




$$
\begin{gathered}
\cdot \frac{\Gamma((\beta+v+\gamma) r+j+1)}{\Gamma((\beta+v+\gamma) r+j+1+v)} \frac{\Gamma((\beta+v+\gamma) r+v+\gamma+j+1)}{\Gamma((\beta+v+\gamma) r+v+\gamma+\beta+j+1+v)} \\
\cdot(x-a)^{\alpha(\beta+v+\gamma) r+\alpha j+\alpha v+\alpha \gamma+\alpha \beta} \\
=\frac{\lambda^{r+1}}{\alpha^{(r+1)(v+\beta)}} C_{\beta, v}(\beta+v+\gamma, j, r) \frac{\Gamma((\beta+v+\gamma) r+j+1)}{\Gamma((\beta+v+\gamma) r+j+1+v)} \\
\cdot \frac{\Gamma((\beta+v+\gamma)(r+1)+j+1-\beta)}{\Gamma((\beta+v+\gamma)(r+1)+j+1+v)}(x-a)^{\alpha(\beta+v+\gamma) r+\alpha j+\alpha v+\alpha \gamma+\alpha \beta} \\
=\frac{\lambda^{r+1}}{\alpha^{(r+1)(v+\beta)}} C_{\beta, v}(\beta+v+\gamma, j, r+1)(x-a)^{\alpha(\beta+v+\gamma)(r+1)+\alpha j} .
\end{gathered}
$$

Thus, equality (25) also holds for the case $r+1$. Obviously, for the given values of parameters $\alpha, \beta, \gamma, v$, for any $i \geq 1$, the inequality $C_{\beta, v}(\beta+v+\gamma, j, i) \neq 0$ is satisfied. The lemma is proved.

$$
\begin{aligned}
& \text { Let } C_{\beta, v}(\beta+v+\gamma, j, 0)=1 \text { and consider functions } \\
& \qquad u_{j}(z)=\sum_{i=0}^{\infty} C_{\beta, v}(\beta+v+\gamma, j, i) z^{i}, j=0,1, \ldots, n-1 .
\end{aligned}
$$

where $z-$ is a complex number.

If in equality (26) $\gamma=0$, then

$$
\begin{gathered}
C_{\beta, v}(\beta+v, j, i)=\prod_{k=0}^{i-1} \frac{\Gamma[(\beta+v) k+j+1]}{\Gamma[(\beta+v) k+j+1+v]} \cdot \frac{\Gamma[(\beta+v)(k+1)+j+1-\beta]}{\Gamma[(\beta+v)(k+1)+j+1]} \\
=\frac{\Gamma(j+1)}{\Gamma[i(\beta+v)+j+1]},
\end{gathered}
$$

and

$$
u_{j}(z)=\sum_{i=0}^{\infty} \frac{\Gamma(j+1)}{\Gamma[i(\beta+v)+j+1]} z^{i}=\Gamma(j+1) E_{\beta+v, j+1}(z) .
$$

Moreover, for $v=0$ the following equality holds:

$$
\begin{gathered}
C_{\beta, 0}(\beta+\gamma, j, i)=\prod_{k=0}^{i-1} \frac{\Gamma[(\beta+\gamma) k+j+1]}{\Gamma[(\beta+\gamma) k+j+1]} \cdot \frac{\Gamma[(\beta+\gamma)(k+1)+j+1-\beta]}{\Gamma[(\beta+\gamma)(k+1)+j+1]} \\
=\prod_{k=0}^{i-1} \frac{\Gamma[(\beta+\gamma)(k+1)+j+1-\beta]}{\Gamma[(\beta+\gamma)(k+1)+j+1]}=\prod_{k=0}^{i-1} \frac{\Gamma[(\beta+\gamma) k+\beta+\gamma+j+1-\beta]}{\Gamma[(\beta+\gamma) k+\beta+\gamma+j+1]} \\
=\prod_{k=0}^{i-1} \frac{\Gamma[\beta((1+\gamma / \beta) k+(\gamma+j) / \beta)+1]}{\Gamma[\beta((1+\gamma / \beta) k+(\gamma+j) / \beta+1)+1]}=c_{i},
\end{gathered}
$$

i.e., these coefficients coincide with the expansion coefficients of the function $E_{\beta, m, l}(z)$ with the indices $m=1+\frac{\gamma}{\beta}, \ell=\frac{\gamma+j}{\beta}$. It was shown in [30] that the coefficients of the function $E_{\beta, m, l}(z)$ satisfy the asymptotic estimate

$$
\frac{c_{i}}{c_{i+1}}=(\beta m i)^{\beta} \rightarrow \infty(i \rightarrow \infty)
$$

Whence it follows that the function $E_{\beta, m, l}(z)$ is an integral function.

Let us introduce the notation $\delta=\beta+\gamma+v$ and rewrite coefficients $C_{\beta, v}(\beta+v+\gamma, j, i)$ as

$$
C_{\beta, v}(\delta, j, i)=\prod_{k=0}^{i-1} \frac{\Gamma[\delta k+j+1]}{\Gamma[\delta k+j+1+v]} \cdot \frac{\Gamma[\delta k+\delta+j+1-\beta]}{\Gamma[\delta k+\delta+j+1]}
$$




$$
=\prod_{k=0}^{i-1} \frac{\Gamma\left[v\left(k \frac{\delta}{v}+\frac{j}{v}\right)+1\right]}{\Gamma\left[v\left(k \frac{\delta}{v}+\frac{j}{v}+1\right)+1\right]} \cdot \frac{\Gamma\left[\beta\left(k \frac{\delta}{\beta}+\frac{\delta+j-\beta}{\beta}\right)+1\right]}{\Gamma\left[\beta\left(k \frac{\delta}{\beta}+\frac{\delta+j-\beta}{\beta}+1\right)+1\right]}, i \geq 1, v>0 .
$$

Further, from the asymptotic estimate

$$
\frac{C_{\beta, v}(\delta, j, i)}{C_{\beta, v}(\delta, j, i+1)}=(\delta i)^{v+\beta} \rightarrow \infty(i \rightarrow \infty),
$$

it follows that $u_{j}(z), j=1,2, \ldots, m$, from equality (27) it also follows that they are integral functions.

Lemma 4 and Proposition 2 imply the following lemma.

Lemma 5. Let $\alpha>0, n-1<\beta \leq n, \gamma, v \geq 0$. Then for all values $j=0,1, \ldots, n-1$ the system of functions (25) is 0-normalized with respect to the pair of operators $\left({ }_{a}^{C \beta} D^{\alpha}, \lambda(x-a)^{\alpha \gamma} \cdot{ }_{a}^{v} J^{\alpha}\right)$ in the domain $x>a$.

Using the main property of normalized systems, we obtain the following assertion.

Theorem 7. Let $\alpha>0, n-1<\beta \leq n, \gamma, v \geq 0$ Then for all values $j=0,1, \ldots, n-1$ the functions

$$
y_{j}(x)=\sum_{i=0}^{\infty} f_{i, j}(x) \equiv(x-a)^{\alpha j} \sum_{i=0}^{\infty} \frac{\lambda^{i}}{\alpha^{i(\beta+v)}} C_{\beta, v}(\beta+v+\gamma, j, i)(x-a)^{\alpha(\beta+v+\gamma) i}
$$

are linearly independent solutions of the homogeneous equation (23) where $y_{j}(t) \in C[a, b]$, ${ }_{a}^{C \beta} D^{\alpha} y_{j}(x) \in C[a, b]$.

Proof. Consider the function

$$
u_{j}(x)=\sum_{i=0}^{\infty} \lambda^{i} C_{\beta, v}(\beta+v+\gamma, j, i)(x-a)^{i}, j=0,1, \ldots, n-1 .
$$

Since function (27) is an integral function, it is obvious that

$$
y_{j}(x)=(x-a)^{\alpha j} u_{j}\left(\lambda \alpha^{-(\beta+v)}(x-a)^{\alpha(\beta+v+\gamma)}\right) \in C[a, b]
$$

and

$$
{ }_{a}^{C \beta} D^{\alpha} y_{j}(x)=\lambda y_{j}(x) \in C[a, b] .
$$

for $j=0,1, \ldots, n-1$. Therefore, functions $y_{j}(x)$ from (28) are solutions to the homogeneous equation (23). The proof of the linear independence of solutions (28) will be shown below in Theorem 11. The theorem is proved.

Further, we will consider a method for constructing a solution to the inhomogeneous equation. Let $f(x) \in C[a, b]$. Then, by the proposition of Lemma 3 , the function $f_{0}(x)=$ ${ }_{a}^{\beta} J^{\alpha} f(x)$ satisfies the equality

$$
L_{1} f_{0}(x)={ }_{a}^{C \beta} D^{\alpha}\left[{ }_{a}^{\beta} J^{\alpha} f\right](x)=f(x) .
$$

Consider the system

$$
f_{i}(x)=\left({ }_{a}^{\beta} J^{\alpha} \cdot \lambda(x-a)^{\alpha \gamma} \cdot{ }_{a}^{v} J^{\alpha}\right)^{i} f_{0}(x) \equiv \lambda^{i}\left({ }_{a}^{\beta} J^{\alpha}(x-a)^{\alpha \gamma} \cdot{ }_{a}^{v} J^{\alpha}\right)^{i} f_{0}(x), i=1,2, \ldots
$$


Lemma 6. Let $f(x) \in C[a, b]$. Then the system of function (29) is $f(x)$-normalized with respect to the pair of operators $\left({ }_{a}^{C \beta} D^{\alpha}, \lambda(x-a)^{\alpha \gamma} \cdot{ }_{a}^{v} J^{\alpha}\right)$ in the domain $x>a$.

Proof. Let $f(x) \in C[a, b]$, then

$$
\left|f_{0}(x)\right|=\left|{ }_{a}^{\beta} J^{\alpha} f(x)\right| \leq\|f\|_{C[a, b]} \underset{a}{\beta} J^{\alpha}(1)(x) \leq\|f\|_{C[a, b]} \frac{1}{\alpha^{\beta}} \frac{\Gamma(1)}{\Gamma(\beta+1)}(x-a)^{\alpha \beta} .
$$

Hence

$$
\left|f_{0}(x)\right| \leq \frac{\|f\|_{C[a, b]}}{\alpha^{\beta}} \frac{\Gamma(1)}{\Gamma(\beta+1)}(x-a)^{\alpha \beta},\left\|f_{0}(x)\right\|_{C[a, b]} \frac{\|f\|_{C[a, b]}}{\alpha^{\beta}} \frac{\Gamma(1)}{\Gamma(\beta+1)}(b-a)^{\alpha \beta} .
$$

Further, we use the notation $M=\frac{\|f\|_{C[a, b]}}{\alpha^{\beta}} \frac{\Gamma(1)}{\Gamma(\beta+1)}$. Then $\left|f_{0}(x)\right| \leq M(x-a)^{\alpha \beta}$ From the latter estimate it follows that

$$
\left|\left({ }_{a}^{\beta} J^{\alpha} \cdot \lambda(x-a)^{\alpha \gamma} \cdot{ }_{a}^{v} J^{\alpha}\right) f_{0}(t)\right| \leq M|\lambda|\left({ }_{a}^{\beta} J^{\alpha} \cdot(x-a)^{\alpha \gamma} \cdot{ }_{a}^{v} J^{\alpha}\right)(x-a)^{\alpha \beta} .
$$

Hence, for any $i \geq 1$ the estimate is valid:

$$
\left|f_{i}(x)\right|=\left|\left({ }_{a}^{\beta} J^{\alpha} \cdot \lambda(x-a)^{\alpha \gamma} \cdot{ }_{a}^{v} J^{\alpha}\right)^{i} f_{0}(x)\right| \leq M|\lambda|^{i}\left({ }_{a}^{\beta} J^{\alpha} \cdot(x-a)^{\alpha \gamma} \cdot{ }_{a}^{v} J^{\alpha}\right)^{i}(x-a)^{\alpha \beta} .
$$

Let us calculate the value of the function $g_{i}(x)=\left({ }_{a}^{\beta} J^{\alpha} \cdot(x-a)^{\alpha \gamma} \cdot{ }_{a}^{v} J^{\alpha}\right)^{i}(x-a)^{\alpha \beta}$. Due to equality (25) we get

$$
g_{i}(x)=\frac{1}{\alpha^{i(\beta+v)}} C_{\beta, v}(\beta+v+\gamma, \beta, i)(x-a)^{\alpha(\beta+v+\gamma) i+\alpha \beta}, i=1,2, \ldots .
$$

Hence, for any $i \geq 1$ the relation $f_{i}(x) \in C[a, b]$ and estimates

$$
\left|f_{i}(x)\right| \leq M \frac{|\lambda|^{i}}{\alpha^{i(\beta+v)}} C_{\beta, v}(\beta+v+\gamma, \beta, i)(x-a)^{\alpha(\beta+v+\gamma) i+\alpha \beta}
$$

are valid. Moreover,

$$
\left({ }_{a}^{\beta} J^{\alpha} \cdot \lambda(x-a)^{\alpha \gamma} \cdot{ }_{a}^{v} J^{\alpha}\right)^{i} f_{0}(x)=\left({ }_{a}^{\beta} J^{\alpha} \cdot \lambda(x-a)^{\alpha \gamma} \cdot{ }_{a}^{v} J^{\alpha}\right) f_{i-1}(x)={ }_{a}^{\beta} J^{\alpha} g(x)
$$

where

$$
g(x)=\lambda(x-a)^{\alpha \gamma} \cdot{ }_{a}^{v} J^{\alpha} f_{i-1}(x) .
$$

As $f_{i-1}(x) \in C[a, b]$, then $g(x)=\lambda(x-a)^{\alpha \gamma} \cdot{ }_{a}^{v} J^{\alpha} f_{i-1}(x)$ also belongs to class $C[a, b]$ and the equality is satisfied:

$$
\begin{gathered}
L_{1} f_{i}(x)={ }_{a}^{C \beta} D^{\alpha}\left({ }_{a}^{\beta} J^{\alpha} \cdot \lambda(x-a)^{\alpha \gamma} \cdot{ }_{a}^{v} J^{\alpha}\right)^{i} f_{0}(x)={ }_{a}^{C \beta} D^{\alpha}\left[{ }_{a}^{\beta} J^{\alpha} g\right](x)=g(x) \\
=\lambda(x-a)^{\alpha \gamma} \cdot{ }_{a}^{v} J^{\alpha} f_{i-1}(x)=L_{2} f_{i-1}(x), i \geq 1 .
\end{gathered}
$$

It is obvious that $L_{1} f_{0}(x)={ }_{a}^{C \beta} D^{\alpha}\left(f_{0}\right)(x)=f(x)$. Thus, in the class of functions $X=C[a, b]$ the equalities

$$
L_{1} f_{0}(x)=f(x), L_{1} f_{i}(x)=L_{2} f_{i-1}(x), i \geq 1
$$

hold, i.e., system (29) is $f$ - normalized with respect to the pair of operators

$$
\left({ }_{a}^{C \beta} D^{\alpha}, \lambda(x-a)^{\alpha \gamma} \cdot{ }_{a}^{v} J^{\alpha}\right)
$$


The lemma is proved.

Theorem 8. Let $f(x) \in C[a, b], f_{0}(x)={ }_{a}^{\beta} J^{\alpha} f(x)$ and function $f_{i}(x), i \geq 1$ are defined by equality (29). Then the function

$$
y_{f}(x)=\sum_{i=0}^{\infty} f_{i}(x)
$$

is a particular solution of equation (23) from the class $C[a, b]$.

Proof. Let us estimate the series (31). By virtue of estimate (30), we have

$$
\left|y_{f}\right| \leq \sum_{i=0}^{\infty}\left|f_{i}(t)\right| \leq M|| f||_{C[0, d]}(x-a)^{\alpha \beta}\left[1+\sum_{i=1}^{\infty}|\lambda|^{i} C_{\beta, v}(\beta+\gamma+v, \beta, i)(x-a)^{i(\beta+\gamma+v)}\right]
$$

As the latter series converges uniformly in the domain $a \leq t \leq b$, the sum of this series and hence the function $y_{f}(t)$ belong to class $C[a, b]$. The theorem is proved.

Let us investigate the representation of function (31) for some special cases of function $f(x)$.

Lemma 7. Let $f(x)=(x-a)^{\alpha \mu}, \mu \geq 0$. Then the particular solution of equation (23) is written as

$$
y_{\mu}(t)=\frac{1}{\alpha^{\beta}} \frac{\Gamma(\mu+1)(x-a)^{\alpha(\mu+\beta)}}{\Gamma(\mu+1+\beta)} \sum_{i=0}^{\infty} \frac{\lambda^{i}}{\alpha^{i(\beta+v)}} C_{\beta, v}(\beta+\gamma+v, \mu+\beta, i)(x-a)^{\alpha i(\beta+\gamma+v)}
$$

Proof. In this case for $f_{i}(x)$ from (29) we get

$$
\begin{aligned}
& f_{i}(x)=\left({ }_{a}^{\beta} J^{\alpha} \cdot \lambda(x-a)^{\alpha \gamma} \cdot v_{a}^{v} J^{\alpha}\right)^{i}{ }_{a}^{\beta} J^{\alpha}(x-a)^{\alpha \mu}=\frac{1}{\alpha^{\beta}} \frac{\Gamma(\mu+1)}{\Gamma(\mu+1+\beta)}\left({ }_{a}^{\beta} J^{\alpha} \cdot \lambda(x-a)^{\alpha \gamma} \cdot v_{a}^{v} J^{\alpha}\right)^{i} \\
& \cdot(x-a)^{\alpha(\mu+\beta)}=\frac{1}{\alpha^{\beta}} \frac{\Gamma(\mu+1)}{\Gamma(\mu+1+\beta)} \frac{1}{\alpha^{i(\beta+v)}} C_{\beta, v}(\beta+\gamma+v, \mu+\beta, i)(x-a)^{\alpha i(\beta+\gamma+v)+\alpha(\mu+\beta)} .
\end{aligned}
$$

The lemma is proved.

This lemma implies the following assertion.

Theorem 9. Let $f(x)=\sum_{j=1}^{p} \lambda_{j}(x-a)^{\mu_{j}}, \mu_{j} \geq 0 \mu_{j}>-1$. Then the particular solution of equation (23) is written as

$$
\begin{aligned}
& y_{f}(t)=\sum_{j=1}^{p} \frac{\lambda_{j} \Gamma\left(\mu_{j}+1\right)(x-a)^{\alpha\left(\beta+\mu_{j}\right)}}{\alpha^{\beta} \Gamma\left(\mu_{j}+1+\beta\right)} \\
& \quad \times \sum_{i=0}^{\infty} \frac{\lambda^{i}}{\alpha^{i(\beta+v)}} C_{\beta, v}\left(\beta+\gamma+v, \mu_{j}+\beta, i\right)(x-a)^{i \alpha(\beta+\gamma+v)} .
\end{aligned}
$$

Remark 4. In case $\alpha=1$ representation (32) of a particular solution of equation (23) coincides with the result of [27].

Further, let us investigate the following Cauchy-type problem

$$
\begin{gathered}
{ }_{a}^{C \beta} D^{\alpha} y(x)=\lambda(x-a)^{\alpha \gamma} \cdot{ }_{a}^{v} J^{\alpha} y(x)+\sum_{j=1}^{p} \lambda_{j}(x-a)^{\alpha \mu_{j}}, \mu_{j} \geq 0, a<x, \\
\left.{ }_{a}^{m} T^{\alpha} y(x)\right|_{x=a}=d_{m}, m=0,1, \ldots, n-1
\end{gathered}
$$


where $d_{k}$ are real numbers. First, let us consider the homogeneous problem (33), (34).

Theorem 10. Let $\lambda_{j}=0, j=1,2, \ldots, p$. Then the solution to the Cauchy problem (33), (34) exists, is unique, and can be represented as

$$
y(x)=\sum_{j=0}^{n-1} \frac{d_{j}}{\alpha^{j} \Gamma(j+1)}(x-a)^{\alpha j} \sum_{i=0}^{\infty} \frac{\lambda^{i}}{\alpha^{i(\beta+v)}} C_{\beta, v}(\beta+v+\gamma, j, i)(x-a)^{\alpha(\beta+v+\gamma) i} .
$$

Proof. Let $\lambda_{j}=0, j=1,2, \ldots, p$. According to Theorem 7 , function $y(x)$ in (35) is a solution to equation (33). Let us show that $y(x)$ satisfies the initial conditions (34). For function $(x-a)^{\alpha j}$ we have

$$
\begin{aligned}
& { }_{a}^{m} T^{\alpha}(x-a)^{\alpha j}=0, m>j,{ }_{a}^{m} T^{\alpha}(x-a)^{\alpha j}=\alpha j(\alpha j-\alpha) \ldots(\alpha j-(m-1) \alpha)(x-a)^{\alpha(j-m)} \\
& =\alpha^{m} j(j-1) \ldots(j-(m-1))(x-a)^{\alpha(j-m)}=\frac{\alpha^{m} \Gamma(j+1)}{\Gamma(j+1-m)}(x-a)^{\alpha(j-m)}, m=1,2 \ldots, j .
\end{aligned}
$$

Then

$$
\lim _{x \rightarrow a}{ }_{a}^{m} T^{\alpha}(x-a)^{\alpha j}=\left\{\begin{array}{l}
\alpha^{m} \Gamma(m+1), m=j \\
0, m<j
\end{array} .\right.
$$

Hence, for function $y(x)$ we get

$$
\left.{ }_{a}^{m} T^{\alpha} y(x)\right|_{x=a}=\lim _{x \rightarrow a}{ }_{a}^{m} T^{\alpha} y(x)=\frac{d_{m}}{\alpha^{m} \Gamma(k+1)} \alpha^{m} \Gamma(k+1)=d_{m} .
$$

The theorem is proved.

From Theorem 10 the following theorem can be derived.

Theorem 11. Functions $y_{j}(t), j=0,1, \ldots, n-1$ from (28) are linearly independent.

Proof. For functions $y_{0}(t), y_{1}(t), \ldots, y_{n-1}(t)$ we introduce an analogue of Wronskian: $W_{\alpha}(x)=$ $\operatorname{det}\left({ }_{a}^{m} T^{\alpha} y_{j}(x)\right)_{m, j=1}^{n-1}, a \leq x \leq b$.

As in the case of the theorem for linear differential equations of order $n$, the following statement can be proved.

Lemma 8. For solutions $y_{1}(t), y_{2}(t), \ldots, y_{m}(t)$ to equations (33) be linearly independent, it is necessary and sufficient that $W_{\alpha}\left(x_{0}\right) \neq 0$ at a point $x_{0} \in[a, b]$.

According to (36) we get $W_{\alpha}(a) \neq 0$ and, hence, according to the lemma, the solutions $y_{1}(t), y_{2}(t), \ldots, y_{m}(t)$ to equation (33) are linearly independent. The theorem is proved.

Theorems 9 and 10 imply the following assertion.

Theorem 12. If $\mu_{j} \geq 0, j=1,2, \ldots, p$, then the solution to the Cauchy problem (33), (34) exists, is unique, and can be represented as

$$
\begin{gathered}
y(x)=\sum_{j=0}^{n-1} \frac{d_{j}}{\alpha^{j} \Gamma(j+1)}(x-a)^{\alpha j} \sum_{i=0}^{\infty} \frac{\lambda^{i}}{\alpha^{i(\beta+v)}} C_{\beta, v}(\beta+v+\gamma, j, i)(x-a)^{\alpha(\beta+v+\gamma) i} \\
+\sum_{j=1}^{p} \frac{\lambda_{j} \Gamma\left(\mu_{j}+1\right)(x-a)^{\alpha\left(\beta+\mu_{j}\right)}}{\alpha^{\beta} \Gamma\left(\mu_{j}+1+\beta\right)} \sum_{i=0}^{\infty} \frac{\lambda^{i}}{\alpha^{i(\beta+v)}} C_{\beta, v}\left(\beta+\gamma+v, \mu_{j}+\beta, i\right)(x-a)^{i \alpha(\beta+\gamma+v)}
\end{gathered}
$$

Remark 5. Similar investigations can be carried out for the equation with the operator ${ }_{a}^{\beta} D^{\alpha}$. 
Author Contributions: Batirkhan Turmetov, Kairat Usmanov and Kulzina Nazarova contributed equally to the conceptualization and the formal analysis of the problem discussed in the paper. All authors have read and agreed to the published version of the manuscript.

Funding: The work was supported by a grant from the Ministry of Science and Education of the Republic of Kazakhstan (grant no.AP08956307).

Institutional Review Board Statement: Not applicable.

Informed Consent Statement: Not applicable.

Data Availability Statement: All the data is present within the manuscript.

Conflicts of Interest: The authors declare no conflict of interest.

\section{References}

1. Hilfer, R. Applications of Fractional Calculus in Physics, 1st ed.; World Scientific: Singapore, 2000. ISBN: 9810234570.

2. Kilbas, A.A.; Srivastava, H.M.;Trujillo, J.J. Theory and Application of Fractional Differential Equations, 1st ed.; Elsevier Science B.V.: Amsterdam, The Netherlands, 2006. ISBN 0444518320;9780444518323.

3. Magin, R.L. Fractional Calculus in Bioengineering, Begell House Publishers: Redding, 2006. ISBN 1567002153;978-1567002157.

4. Podlubny, I. Fractional Differential Equations, 1st ed.; Academic Press: San Diego, CA, USA, 1999. ISBN 558840-2.

5. Samko, S.G.; Kilbas, A.A.; Marichev, O.I. Fractional Integrals and Derivatives: Theory and Applications, 1st ed.;CRC Press: Switzerland, 1993. ISBN 2881248640;978-2881248641.

6. Baleanu, D.; Fernandez, A. On Fractional Operators and Their Classifications. Mathematics. 2019, 7, 1-10.

7. Bargamadi, E.; Torkzadeh, L.; Nouri, K.; Jajarmi, A . Solving a system of fractional-order volterra-fredholm integro-differential equations with weakly singular kernels via the second Chebyshev wavelets method. Fractal Fract. 2021, 5, 70. P.1-14.

8. Jarad, F.; Ugurlu, E.; Abdeljawad, T.; Baleanu, D. On a new class of fractional operators. Advances in Difference Equations. 2017, $247,1-16$.

9. Katugampola, U.N. New approach to generalized fractional integral. Appl. Math. Comput. 2011, 218, 860-865.

10. Hilfer, R.; Luchko, Yu.; Tomovski, Z. Operational method for the solution of fractional differential equations with generalized Riemann-Liouville fractional derivatives. Fract. Calc. Appl. Anal 2009, 12, 299-318.

11. Luchko, Yu. Operational method in fractional calculus. Fract. Calc.Appl. Anal. 1999, 2, 463-488.

12. Luchko, Yu.; Gorenflo, R. An operational method for solving fractional differential equations. Acta Mathematica Vietnamica 1999, 24, 207-234.

13. Luchko, Yu.; Srivastava, H.M. The exact solution of certain differential equations of fractional order by using operational calculus. Comput. Math. Appl. 1995, 29, 73-85.

14. Luchko, Yu. Operational calculus for the general fractional derivative and its applications. Fractional Calculus and Applied Analysis 2021, 24,338-375.

15. Luchko, Yu. General Fractional Integrals and Derivatives with the Sonine Kernels. Mathematics 2021, 9, 1-17.

16. Kim, M.H.; Ri, G.C.; Hyong-Chol, O. Operational method for solving multi-term fractional differential equations with the generalized fractional derivatives. Fract. Calc. Appl. Anal. 2014, 17, 79-95.

17. Pskhu, A.V. Initial-value problem for a linear ordinary differentialequation of noninteger order. Sbornik: Mathematics 2011, 202, 571-582.

18. Cheng, J.Fa.; Chu, Y.M. Solution to the linear fractional differentialequation using Adomian decomposition method. Mathematical Problems in Engineering 2011, 1, 1-14.

19. Hu, Y.; Luo, Y.; Lu, Z. Analytical solution of the linear fractional differential equation by Adomian decomposition method. J. of Computational and Applied Mathematics 2008, 215, 220-229.

20. Karachik, V.V. Normalized system of functions with respect to the Laplace operator and its applications. Journal of Mathematical Analysis and Application, 2003, 287, 577-592.

21. Karachik, V.V. Method for constructing solutions of linear ordinary differential equations with constant coefficients. Computational Mathematics and Mathematical Physics, 2012, 52, 219-234.

22. Liu, L.; Ren, G.B. Normalized system for wave and Dunkl operators.Taiwanese Journal of Mathematics, 2007, 14, 675-683.

23. Yuan, H.F.; Karachik ,V.V. Dunkl-Poisson Equation and Related Equations in Superspace.Mathematical Modelling and Analysis, 2015, 20, , 768-781.

24. Ashurov, R.R.; Cabada, A.; Turmetov, B.Kh. Operator method for construction of solutions of linear fractional differential equations with constant coefficients. Fractional Calculus and Applied Analysis, 2016. 19, $229-251$.

25. Turmetov B. On Certain Operator Method for Solving Differential Equations. Filomat 2017, 31, 4275-4286.

26. Turmetov B. Construction of explicit solutions of fractional integral-differential equations of Volterra type. AIP Conference Proceedings 2017, 1880, 1-6.

27. Turmetov, B.Kh. On a method for constructing a solution of integral - differential equations of fractional order Electronic Journal of Qualitative Theory of Differential Equations 2018,25, 1-14. 
28. Kilbas, A.A.; Saigo, M. On solution of integral equation of Abel-Volterra type. Differential and Integral Equations 1995, 8, 993-1011.

29. Kilbas, A.A.; Saigo ,M. Fractional integral and derivatives of Mittag-Leffler type function.Dokl. Akad. Nauk Belarus 1995, 39, 22-26.

30. Kilbas, A.A.; Saigo, M. On Mittag-Leffler type function, fractional calculus operators andsolutions of integral equations. Integral Transform. Spec. Funct. 1996, 4, 355-370.

31. Boudabsa, L.; Simon, T. Some Properties of the Kilbas-Saigo Function. Mathematics 2021, 9, 1-24.

32. Kilbas A.A., Saigo M., Solution in closed form of a class of linear differential equations of fractional order Differ. Uravneniya 1997, 33, 95-204 